\title{
Parameter study of synchroniser mechanisms applied to dual clutch transmissions
}

\author{
Paul Walker* \\ Faculty of Engineering and IT, \\ University of Technology, Sydney \\ 15 Broadway, Ultimo, NSW, 2007, Australia \\ email: paul.walker@uts.edu.au \\ *Corresponding author
}

\author{
Nong Zhang \\ Faculty of Engineering and IT, \\ University of Technology, Sydney \\ 15 Broadway, Ultimo, NSW, 2007, Australia \\ email: nzhang@uts.edu.au
}

\section{Biographical Notes:}

Paul D. Walker received his BE with Honours in mechanical engineering from the University of Technology, Sydney in Australia in 2007. He is currently pursuing a Doctorate of Philosophy at the University of Technology, Sydney in Powertrain Dynamics and is expected to graduate in July of 2011. His current research interests include vibration analysis, hydraulic control systems modelling, vehicle powertrain and powertrain component modelling, particularly the dual clutch transmission and synchroniser mechanisms, and the modelling, simulation, and control of dynamic systems. 
Prof Zhang received his BE degree in 1982 from Northeastern University, ME in 1984 from Shanghai Jiao Tong University, China, and $\mathrm{PhD}$ in 1989 from the University of Tokyo, Japan. He worked at several universities in China, Japan, USA and Australia respectively before joining the Faculty of Engineering of the University of Technology, Sydney in 1995. Since 2009, he has been Professor of Mechanical Engineering, at School of Electrical, Mechanical and Mechatronic Systems. For more than 20 years, Prof Zhang has been involved in research in areas of dynamics and control of automotive systems including powertrains with various types of transmissions, hybrid propulsion systems, vehicle dynamics, passive and active suspensions; and mechanical vibration including experimental modal analysis, rotor dynamics, cold rolling mill chatter and machine condition monitoring.

\footnotetext{
Abstract

The modelling, simulation and analysis of a synchromesh synchroniser as a component of a wet dual clutch transmission is presented in this paper. The synchroniser mechanism is used to match gear speeds and interlock the target gear with the shaft prior to the commencement of gear shifting. Engagement of the synchroniser is modelled as a rigid body system, with consideration to the development of a detailed drag torque model to establish its variation over the process. The implication of choice of design parameters for the synchroniser is only full realised once the speed dependence of drag torque is established. To further develop an understanding of both the influence of design parameters and constraints for the mechanism and to demonstrate the influence of drag
} 
torque over a wide range of operating conditions, dimensionless equivalent torques are developed as a function of cone clutch design radius and hydraulic load. Parameter studies are performed using the model to demonstrate the validity of using dimensionless equivalent cone and indexing torques from a design perspective.

The obtained results suggest that the time duration of speed synchronisation can be significantly reduced through the application of large friction coefficient or smaller cone angles, but there is a limit to be maintained in order to friction lock of the cone. The friction coefficient variation in the indexing and blocking chamfers has minor influence on the engagement of the mechanism, whilst the use of acute chamfer angles will reduce the engagement time without significantly increasing the likelihood of early unblocking of the mechanism.

Keywords: Synchroniser, Synchromesh, Drag Torque, Dual Clutch Transmission, DCT, Parameter Study

\section{Nomenclature}

$\alpha$ - cone angle

$\beta$ - Chamfer angle

$\delta$ - Chamfer relative displacement

$\dot{\theta}_{\mathrm{S}}-$ Cone relative speed and

$\ddot{\theta}_{F W}$ - Freewheeling component acceleration

$\ddot{\theta}_{R}-$ Ring acceleration

$\mu-$ transmission fluid viscosity 


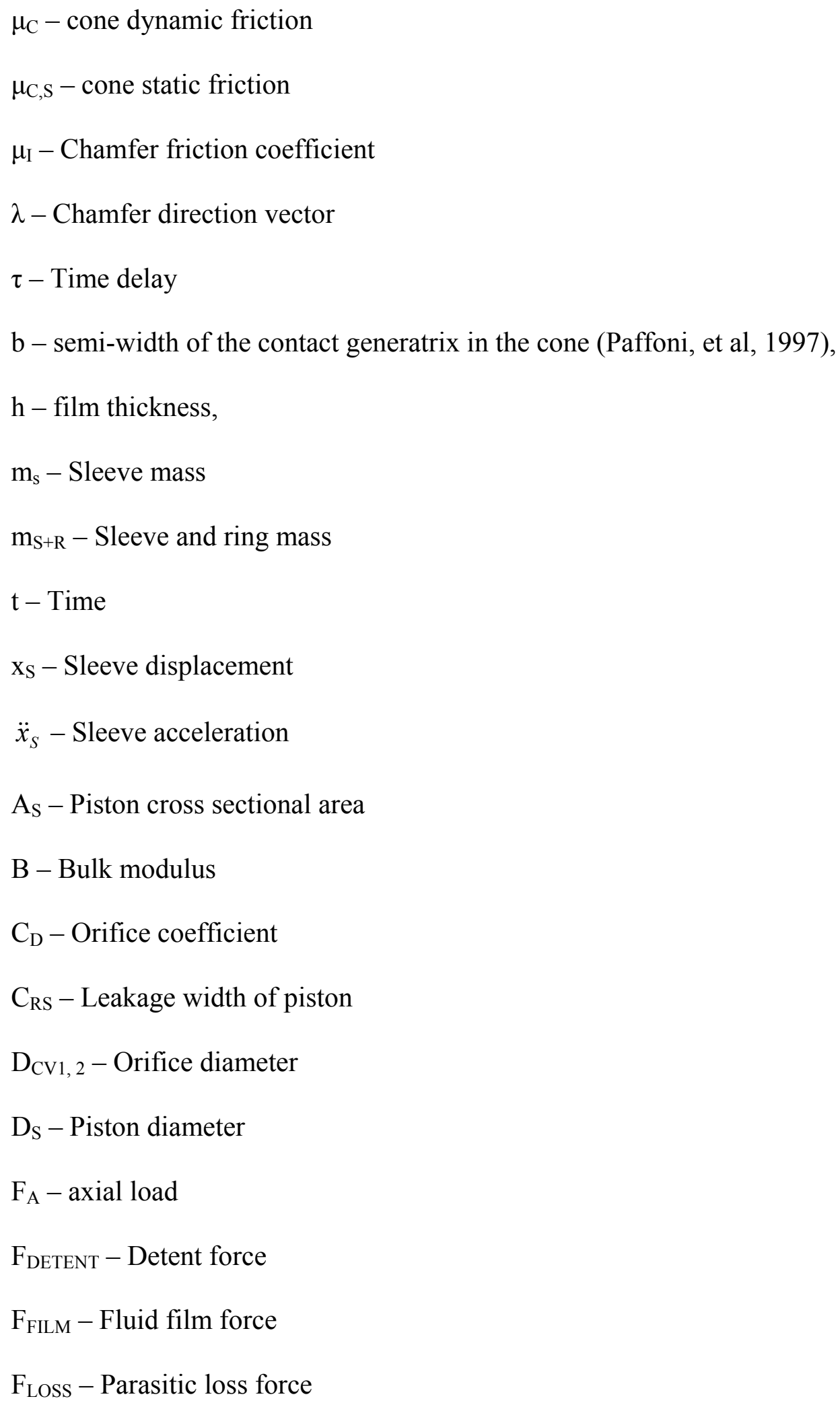


$\mathrm{F}_{\mathrm{SLD}}$ - Sliding force of sleeve over ring during second displacement

$\mathrm{F}_{\mathrm{SLI}}-$ Sliding force of the sleeve over the hub chamfers during indexing

$\mathrm{I}_{\mathrm{FW}}-$ reflected freewheeling gear inertia

$I_{R}-$ Ring Inertia

$\mathrm{P}_{\mathrm{CV} 1}-$ Control volume 1 pressure (Fig 1)

$\mathrm{P}_{\mathrm{CV} 2}-$ Control volume 2 pressure

$\mathrm{P}_{\mathrm{EX}}-$ Exhaust pressure

$\mathrm{P}_{\mathrm{IN}}-$ Input pressure

$\mathrm{P}_{\mathrm{S}}-$ Solenoid output pressure

Q - Flow rate

$\mathrm{R}_{\mathrm{C}}-$ mean cone radius

$\mathrm{R}_{\mathrm{I}}-$ Chamfer pitch radius

$\mathrm{T}_{\mathrm{B}}-$ Blocking torque

$\mathrm{T}_{\mathrm{C}}-$ cone torque

$T_{D}-$ Drag torque

$\mathrm{T}_{\mathrm{I}}-$ Indexing torque

V - Volume

\section{Introduction}

Recent development of the dual clutch transmission (DCT) as a modern vehicle transmission system with the capacity for both power on shifting and improved efficiencies has required the adaptation of conventional manual transmission components to an automated system. Power on shifting, where clutch release and engagement is 
performed simultaneously, differentiates the synchronisation process from application in either manual or automated manual transmission systems. Simulation and modelling of the synchroniser mechanism with respect to the DCT is thus required to evaluate the influence of the transmission, and identify areas where potential performance improvements may be gained.

The role of transmission architecture is particularly significant in the control and actuation of the synchroniser mechanism coupled with a DCT. Key aspects that must be considered in the synchroniser model are the influence of transmission components on the reflected gear inertia, the net drag acting on the synchroniser, and the actuation method, in this instance an electro-hydraulic system not dissimilar to an automated manual transmission (AMT) but very different to the driver actuation in conventional manual transmissions (MT). The model must therefore reflect accurately the detailed characteristics of the DCT that differentiate this transmission from MTs or AMTs.

Common practice when modelling the shift control of a DCT is to simplify the gear synchronisation component of shifting. Models such as Lei, Wang \& Ge (2007) or Kirschstein (2007) ignore the mechanism entirely as it is not considered as critical to the investigation. Zhang, et al, (2005) and Kulkarni, Shim \& Zhang (2007) provide a simple power switch to simulate the actuation of the synchroniser mechanism during shifting. This is however limited by the lack of time delay in the simulations to approximate the delay between shift detection, completion of synchronisation, and the transmission being ready for shift. While the DCT model presented by Goetz (2005) only considers a cone clutch model of the mechanism to simulate actuation; this does not consider additional process steps or evaluate the effectiveness of the synchroniser mechanism for the process. 
Razzaki (2007) has previously conducted investigations into the design of synchronisers for DCT's. In this it is hinted at the importance of simulating numerically accurate drag torque, particularly its importance for selecting both cone friction coefficient and chamfer angle. There is no detailed characterisation of how drag torque affects the synchronisation process as a whole, or for individual sub-processes. Theory presented by Razzacki (2004), Socin \& Walters (1968) and Abdel-Halim, et al, (2000) suggests that drag torque plays a significant role in several key stages of synchronisation. This includes the maintaining of ring blocking during synchronisation, determining if desynchronisation occurs post unblocking of the ring, as well as the overall process duration. In design literature, such as Lechner \& Naunheimer (1999), it is proposed that constant drag torques can be applied to equations for designing various synchroniser parameters, however speed and load dependent models can be deployed, such as Dogan (2004). The incorrect estimation of both the numerical quantity of this drag and how it is applied to the synchroniser can lead to failure through clash, block out or other modes (Socin \& Walters 1968).

In simulations of synchroniser actuation Kim, et al, (2003) and Kelly \& Kent (2000) target the shift force responses for manual transmissions, including modelling linkage, transmission and driveline. Hoshino (1999) models the synchroniser mechanism in detail including both synchronisation and meshing phases of actuation, clearly demonstrating that the hub indexing phase is highly dependent on the initial alignment of chamfer points, as well as actuation force as derived from the sleeve speed. Liu and Tseng (2007) take this analysis further when applying the Taguchi method to improve the overall process and demonstrating the response of the mechanism to a range of variables 
including friction, cone and chamfer angle, and load. Additional work by Lovas, et al, (2006) investigates the role played by lubrication on both cone and indexing friction coefficients, and the source of the double bump phenomenon experienced as two peak forces required by the driver during shifting. In this paper emphasis is placed on the locking of the ring onto the hub as it is heated and cooled during synchronisation.

Following this introduction in section (1), This paper is divided into sections presenting (2) modelling of the synchroniser mechanism and control system throughout the actuation process. (3) A typical engagement using the presented model so as to relate the described process back to results. Followed by (4) a detailed description of how drag torque was modelled for the DCT architecture. In (5) dimensionless characteristic torques are presented to provide a method for selecting synchroniser parameters. Then a parameter study is carried out to compare major components in (6), before concluding remarks in (7).

\section{Synchroniser system modelling}

\subsection{Synchroniser mechanism and control system}

The synchroniser mechanism in the DCT and a model for the hydraulic control system are shown in Figures 1 and 2. The system being studied consists of five main components:

a) The actuator mechanisms, in this case a hydraulic control unit (Fig. 1);

b) Shaft and saddle, the gear rotates freely on the shaft, and the saddle is the mounting for the sleeve, with motion restricted to axial translation (Fig. 2); 
c) Sleeve, which is the primary moving part actuated by the hydraulic unit and engages both ring and hub with internally cut splines with chamfered tips (Fig. 2);

d) Ring, which comprises of the outer friction surface of cone clutch and blocking chamfers that match up the sleeve chamfers (Fig. 2);

e) Hub, consisting of the inner cone clutch friction surface and indexing chamfers, which again mate with the sleeve chamfered splines (Fig. 2).

\subsection{Hydraulic control system model}

The development of models for hydraulic power systems is very well established, and by applying methodologies presented by Manring (2005) and Stringer (1976) both Watechagit \& Srivivasan (2003) and Jeyakumaran \& Zhang (2005) have been able to successfully demonstrate the accuracy of such methods. Following a similar process, two separate control volumes are set for the hydraulic unit, as shown in Figure 1.

As high flow on/off solenoids are used to actuate the mechanism it is possible to assume that the flow into each control volume when actuated is similar to a step input with time delay, thus the actuating pressure is modelled thus:

$$
P_{S}= \begin{cases}P_{E X} & \text { Idle } \\ P_{I N}\left(1-e^{(-t / \tau)}\right) & \text { Engaged }\end{cases}
$$

To determine the pressure in each control volume the net flow must be determined. This is a function of input flow through the orifice, leakage flow around the piston head, change to the control volume size, and compressibility of the hydraulic fluid. Using the compressibility equation, the net flow equation can be arranged such that the conservation of flow can be integrated to determine the local pressure in each control 
volume; these equations are derived in greater detail Appendix 1. For both these control volumes in Figure 1 the pressure equations are presented below:

$$
\begin{aligned}
& P_{C V 1}=\int \frac{B}{V_{0}+d V}\left(C_{D} \pi \frac{D_{C V 1}^{2}}{4} \sqrt{P_{S}-P_{C V 1}}-A_{S} \dot{x}_{S}-C_{D} \pi D_{S} c_{R S} \sqrt{P_{C V 1}-P_{E X}}\right) d t \\
& P_{C V 2}=\int \frac{B}{V_{0}+d V}\left(C_{D} \pi \frac{D_{C V 2}^{2}}{4} \sqrt{P_{S}-P_{C V 2}}+A_{S} \dot{x}_{S}-C_{D} \pi D_{S} c_{R S} \sqrt{P_{C V 2}-P_{E X}}\right) d t
\end{aligned}
$$

During the actuation one volume is pressurised, while the second volume acts as a damper as it sits idle. Numerical solutions to equations 2 and 3 make it possible to determine the transient pressure conditions during the actuation of the synchroniser mechanism. Changes in volume and its rate of change are derived from the analysis of the motion of the mechanism as a reaction to the input hydraulics.

\subsection{Synchroniser mechanism model}

In conjunction with a description of the synchronisation process, a highly nonlinear model of the actuation process is presented below. It was proposed to divide the process of synchroniser engagement into as many as 11 separate phases (Lovas, et al, 2006, Liu \& Tseng, 2007). Though this can accurately describe the overall synchronisation process it does not necessarily define the interrelation between individual phases accurately.

To begin the modelling process several simplifying assumptions are made. First, as the vehicle inertia is much greater than the inertia of the synchroniser mechanism and reflected inertia of the target gears the dynamic model can ignore the variation in vehicle dynamics as a result of synchroniser engagement. Secondly, the influence of temperature variation on the mechanism is ignored as the engagement time is very short, simplifying 
computational demand. Non-linearity in gear backlash and the vibration component of the transmission model are also ignored. Thus the model simplifies to multiple rigid body system with the bodies of interest being the synchroniser sleeve, synchroniser ring, and target gear, which includes a reflected inertia of all components connected to the gear.

The three principle torques acting on the mechanism to define the actuation process are cone clutch torque, blocking/indexing torques, and drag torque. Cone clutch torque is derived as a function of cone angle, friction coefficient, operating radius, and load. The piecewise cone torque also includes a viscous component, as described by Paffoni, et al, (1997), dynamic friction, and the static friction torque, limited by the static coefficient of friction, and is presented below:

$$
T_{C}= \begin{cases}4 \pi \mu R_{C}^{3} b \frac{\dot{\theta}_{S}}{h} & x_{S}<2 \\ \frac{\mu_{D} F_{A} R_{C}}{\sin \alpha} & x_{S} \geq 2, \dot{\theta} \neq 0 \\ T_{D}+I_{F W} \ddot{\theta}_{F W} & x_{S} \geq 2, \dot{\theta}=0 \\ \frac{\mu_{C, S} F_{A} R_{C}}{\sin \alpha} & x_{S} \geq 2, \dot{\theta}=0, T_{D}>T_{C}\end{cases}
$$

Here, if $\mathrm{x}_{\mathrm{s}}<2$ there is viscous contact in the cone clutch. As the sleeve moves forward, $x_{s} \geq 2$, at contact in the cone dynamic friction ensues according to the second component. This is limited by the static friction in the cone once synchronisation is completed, comprising of the net torque at the cone with a static friction limit.

The blocking chamfer torque is a function of chamfer angle, chamfer friction, operating radius, and applied load:

$$
T_{B}=F_{A} R_{I} \frac{1-\mu_{I} \tan \beta}{\mu_{I}+\tan \beta}
$$


The equation (5) above can be used for blocking torque, however for the more complicated indexing torque a variation is required that takes into account relative motion of friction surfaces, and torque direction resulting from contact flank, based on Hoshino's work (1999), where if the contact flank of the chamfer, $\lambda$, produces a torque opposing nominal gear speed a negative torque will result:

$$
T_{I}= \begin{cases}F_{A} R_{I} \frac{1-\mu_{I} \tan \beta}{\mu_{I}+\tan \beta} & \lambda+v e, \dot{x}+v e \\ F_{A} R_{I} \frac{1+\mu_{I} \tan \beta}{\mu_{I}-\tan \beta} & \lambda+v e, \dot{x}-v e \\ -F_{A} R_{I} \frac{1+\mu_{I} \tan \beta}{\mu_{I}-\tan \beta} & \lambda-v e, \dot{x}+v e \\ -F_{A} R_{I} \frac{1-\mu_{I} \tan \beta}{\mu_{I}+\tan \beta} & \lambda-v e, \dot{x}-v e\end{cases}
$$

The first phase of the synchronisation process is the push through of the sleeve and ring to friction contact of the cone clutch, see Figure 3 (a) for applied forces. It begins with a build up of pressure in the hydraulic cylinder, which increases until the breakthrough load is achieved, where there is enough force on the sleeve to push past the detents that maintain neutral position, and ends with dry friction contact of the cone clutch. Initially, oil squeezing in the cone resists the motion, and viscous friction in the cone partially realigns the ring to the blocking position. During this stage high viscosity of the transmission fluid or poor design of cone can lead to reduced dynamic friction coefficient, ultimately resulting in the failure to maintain blocking during speed synchronisation. The equations of motion for sleeve and target gear are:

$$
\begin{aligned}
& m_{S+R} \ddot{x}_{S}=P_{C V 1} A_{S}-P_{C V 2} A_{S}-F_{D E T E N T}-F_{F I L M}-F_{\text {LOSS }} \\
& I_{R} \ddot{\theta}_{R}=-T_{C}
\end{aligned}
$$




$$
I_{F W} \ddot{\theta}_{F W}=T_{C}+T_{D}
$$

At the end of this stage there is initial friction contact in the active cone clutch, and the ring is positioned at its maximum rotational displacement in the sleeve blocking position. Now speed synchronisation of the target gear with the shaft and sleeve begins, in the first instance of contact, assuming that there has only been partial realignment of the ring previously, the ring is rotated into the complete blocking position with the sleeve, and is held here as long as cone torque exceeds the torque generated in ring blocking chamfers. If the cone torque is lower than the blocking torque then the ring will be forced to the neutral position and clash engagement of sleeve and indexing chamfers will transpire. This can occur if oil wiping of the cone friction surfaces is insufficient in the previous stage and the friction coefficient is too low. Synchronisation is described using equation 9 and the second component of the cone torque model, as long as the blocking torque inequality holds. Additionally, if the drag torque exceeds cone torque then there will be failure to synchronise speeds, thus arriving at the inequality in equation 10 .

$$
T_{D} \leq T_{I} \leq T_{C}
$$

Once this synchronisation has completed or there is failure of the ring to maintain the blocking condition, unblocking of the ring commences, shown in Figure 3 (b). If synchronisation is successful, the combined ring and target gear are rotated to align sleeve chamfers with blocking chamfers. However if the clash failure occurs as described previously only the ring is realigned. In any case the realignment is controlled by the blocking torque, and enables the resumption of sleeve displacement. The static cone friction maintains the locking of the cone, but if the drag torque exceeds blocking torque then there will be block out of the sleeve through chamfer lock. Equation 14 
describes the limitation to torque balancing, and it is important to note that for proper actuation static friction in the cone clutch should be maintained during the unblocking of the ring.

$$
\begin{aligned}
& m_{S} \ddot{x}_{S}=P_{C V 1} A_{S}-P_{C V 2} A_{S}-F_{S L}-F_{L O S S} \\
& F_{S L}=\frac{\tan \beta\left(T_{D}+\left(I_{F W}+I_{R}\right) \ddot{\theta}_{F W}\right)}{R_{I}} \\
& \left(I_{F W}+I_{R}\right) \ddot{\theta}_{F W}=T_{I}+T_{D} \\
& T_{D}<T_{I}>T_{C}
\end{aligned}
$$

Post ring unblocking the second displacement starts as the sleeve moves from contact with the blocking chamfers and ends with contact on the indexing chamfers, see Figure 3 (c). Friction contact between the sides of the ring chamfers, and sleeve splines provides resistance for the displacement of the sleeve, and the only load on the cone to maintain friction lock between ring and target gear, see equation 16 for $\mathrm{F}_{\text {SLD. }}$. The use of design error angle and friction come limits similar to those discribed by Vyrabov (1991) in the cone prevents self locking of the mechanism, thus, with high drag torque and low cone torque a differential speed will be reintroduced at the cone. This can be an issue if the relative speed is too high, and can lead to partial clash failure in the next stage; however it is desirable for multi-cone synchronisers as high cone torque can be difficult to overcome during indexing. Though other authors, Lovas, et al, (2006), for example use different methodologies to assume the cone locks in the general case, and can be true for low drag torque situations present in dry clutches, the wet clutch DCT is likely to develop some form of slip in the cone clutch during this stage that can negatively or 
positively influence the engagement outcomes, as it has been demonstrated in Walker, et $a l,(2009)$.

$$
\begin{aligned}
& m_{S} \ddot{x}_{S}=P_{C V 1} A_{S}-P_{C V 2} A_{S}-F_{S L D}-F_{L O S S} \\
& F_{S L D}=\frac{\mu_{R} T_{D}}{R_{I}} \\
& T_{C}=\frac{\mu_{R} T_{D}}{R_{I}} \times \frac{\mu_{C, S} R_{C}}{\sin \alpha} \\
& \left(I_{F W}\right) \ddot{\theta}_{F W}=T_{C}+T_{D} \\
& T_{D} \leq T_{C}
\end{aligned}
$$

Upon contact between sleeve and indexing chamfers the motion of the sleeve is restricted by the realignment of chamfers, with four possible alignments emulated in equation 6. Ideal alignment results in sleeve passing straight through, incompatible alignment with tip-on-tip chamfer contact, positive torque alignment, with indexing torque and drag torque having the same direction, and negative alignment, where indexing and drag torques having opposing direction, this result is demonstrated by Liu \& Tseng (2007). Additionally, momentum built up from restart of slip in the previous stage must be included, combining with the torques to result in a complex variation in motion of the mechanism that leads to the final engagement of the mechanism. Equations of motion result from Figure $3(d)$.

$$
\begin{aligned}
& m_{S} \ddot{x}_{S}=P_{C V 1} A_{S}-P_{C V 2} A_{S}-F_{S L I}-F_{L O S S} \\
& \left(I_{F W}\right) \ddot{\theta}_{F W}=T_{I}+T_{C}+T_{D} \\
& F_{S L I}=\frac{\tan \beta\left(T_{D}+I_{F R} \ddot{\theta}_{F W}\right)}{R_{I}}
\end{aligned}
$$




\section{Typical engagement of synchroniser}

To demonstrate the process of engagement described above a typical engagement is presented. For this purpose the synchronisation of fourth gear is presented with fifth gear engaged to demonstrate a downshift results displaying sleeve displacement and hydraulic pressure outputs are shown in Figures 4 and 5. It is assumed that the synchroniser is at an initial steady state speed sourced from the combined drag torques acting on the freewheeling target gear.

Demonstrated in Figure 4 is the detailed sleeve displacement during the synchroniser's engagement and response of the hydraulic system reflected in Figure 5. These results are consistent with the restriction of sleeve motion through reactive forces generated by coupling with the target gear. During the initial displacement the pressure rises to counter the oil squeezing and detent loads before engaging blocking chamfers, which results in reduced rate of pressure increase as the cylinder volume expands. During ring blocking and synchronisation, pressurisation continues inline with the solenoid's time delayed output with no sleeve motion (Figure 5). But as the cylinder again expands during ring unblocking, the sleeve moves forward restricted by the net blocking torques, and pressure is released. From here the second unrestrained displacement occurs and the sleeve pushes forward with limited resistance until there is contact with the hub chamfers. Pressure increases rapidly at the contact point and then decreases as the chamfers are realigned and the cylinder continues to expand, until it finally reaches the fully displaced position. 
Consistent with driver controlled manual transmission models there are two obvious bump phases linked to the process engagement in Figure 4. First, at the beginning of the ring blocking and synchronisation stage, and the second when a similar result is achieved as the sleeve engages the hub chamfers for hub indexing. These are observed as step increases in hydraulic pressure and should be considered a response to the contact engagement between chamfers between both the sleeve and ring, and sleeve and indexing chamfers. This contact is comparable to the "double bump" phenomenon or "second load" discussed by Kim, et al, (2003) and Lovas, et al, (2006) in manual transmission simulations.

\section{Drag torque modelling}

Drag torques are speed and torque dependent losses in the transmission that influence the engagement of the synchroniser. This section identifies popular literature used for simulating these losses. A simple model of drag is built using this literature to represent these drag torques, and emulate how it varies over the synchronisation process. Drag torque is modelled using multiple sources and compounded to a reflected loss acting at the target gear to demonstrate the capacity for the cone and chamfer torques to overcome resistances acting on the mechanism.

The fundamental method that is applied in design literature is deemed appropriate for design and modelling transmission systems, where values are assumed for the developed drag, usually as a percentage of the total torque. As another example, Lechner \& Naunheimer (1999) advise that drag can typically be assumed to be of the order of 2Nm when designing a synchroniser mechanism. Lovas (2006) identifies the modelling 
of bearings, seals, and oil churning as part of the synchroniser modelling, but provides only minimal detail on the impact of this torque on the mechanism. Similarly, Dogan (2004) includes compression torque and synchroniser shear for a dip lubricated transmission. The alternative method is to develop detailed models of the various sources of drag torque. Such methods have been attempted for spur gear pairs by Anderson \& Lowenthal (1980), for helical gears by Changenet, Oviedo-Marlot \& Velex (2006) and Heingartner \& Mba (2005), and is suggested by Razzacki \& Hottenstein (2007) as appropriate for synchroniser mechanisms. A typically drag is broken down into sources such as bearing losses, gear tooth friction, windage and churning of the gear in fluids, and shear in the wet clutch for a spray lubricated transmission.

The layout of the DCT requires two separate models that must be developed to account for the different speeds. The absolute drag model, using the actual shaft speed, uses the bearing drag equations from Harris (1969), widely established as the fundamental basis for determining bearing losses. Tooth friction is developed from Changenet and Velex (2007), whilst gear windage is based on the fluid dynamics model presented by Diab et al (2004). Dimensionless models are avoided here due to questions raised in Eastwick \& Johnson (2008) about validation of this type of model. The relative drag model is based on the differential speed of the two clutches. Again Harris (1969) is used for bearing losses. Additionally, losses developed in the concentrically located shafts are modelled as Couette flow, and can be simulated using the equation presented by Schilchting (1979) for concentrically aligned rotating cylinders. Finally the resistance generated by the wet multi-plate clutch is modelled based on the theory 
presented by Yuan et al (2007), where effects of mass flow, centrifugal force, and capillary action are used to account for the reduction of drag at high speed.

Such numerical models are very useful for predicting the speed and load dependent drag torque generated in the synchroniser mechanism. In the steady state the idle synchroniser will arrive at a speed that minimises drag torque. Consequently, a maximum drag is achieved as the hub matches speeds with the sleeve. These results were able to demonstrate that the drag associated with slipping speed of the clutch mechanism, particularly for wet clutches, generates a reversal of the overall drag which has the potential to negatively affect the actuation of the mechanism.

\section{Torque characteristic equations}

Effectively, the synchroniser mechanism actuation is defined with the use of three torques; drag torque, cone clutch torque, and indexing torque. The drag torque is not well understood and despite this there have been many attempts to model drag in various forms as applied to transmission systems. The effect of drag torque on the simulation and control of synchroniser mechanisms needs to be investigated. To evaluate the design, cone and indexing, and applied drag torques on the mechanism in a more general manner one can consider the development of pseudo dimensionless groups of torques by dividing unique torque equations by the applied load, $\mathrm{F}_{\mathrm{A}}$, and a radial counterpart $\mathrm{R}$. As to whether this is the operating radii of cone or chamfer or an alternate radius is used, evaluation of the effect of drag is required. With respect to the cone clutch synchronisation time is evaluated from the target gear inertias, initial and final speeds, and applied loads as: 


$$
t_{s}=\frac{\left(\dot{\theta}_{S, f}-\dot{\theta}_{S, i}\right) I_{F W}}{T_{C} \pm T_{D}}
$$

As $T_{D}$ approaches $T_{C}$, $t_{S}$ approaches infinity, or if $\left|T_{D}\right| \geq\left|T_{C}\right|$ synchronisation is not possible. Alternatively for unblocking of the synchroniser ring consideration of the requirements to move from the blocking to neutral position must be evaluated. See Appendix 2 for detailed derivation of equation, unblocking time can be derived as:

$$
t_{B}=\sqrt{\frac{2 \delta I_{F W}}{T_{D}+T_{B}}}
$$

Thus, as $T_{D}$ approaches $T_{B}, t_{B}$ approaches the square root of infinity, thence block out occurs if $T_{D} \geq T_{B}$. In terms of indexing, the drag torque will only affect the direction of rotation, and only if $T_{D}=T_{I}$ will there be an indexing issue, thus blocking is given a higher priority as a reference torque than its indexing counterpart. Additionally, consideration of the torque inequality $T_{C} \geq T_{B}$ during speed synchronisation to prevent early unblocking of the mechanism and subsequent failure is required.

To fully realise the application and variation of the cone and indexing torques in the design of synchroniser mechanisms these two torque equations, 5 and 6 , can be defined as dimensionless quantities. The definition of torque inequalities in equations 10 , 14 and 19 suggests cone friction torque is the pertinent control variable. Thus, as mentioned at earlier, cone end indexing torques are modified to dimensionless variables with cone mean radius $\mathrm{R}_{\mathrm{C}}$. These are defined as:

$$
\Pi_{C}=\frac{T_{C}}{F_{A} R_{C}}=\frac{\mu_{C}}{\sin \alpha}
$$

And: 


$$
\Pi_{I}=\frac{T_{I}}{F_{A} R_{C}}=\frac{\left(1-\mu_{I} \tan \beta\right)}{\left(\mu_{I}+\tan \beta\right)} \cdot \frac{R_{I}}{R_{C}}
$$

If the radius ratio is maintained as a constant the two dimensionless groups are defined by the design angles and friction coefficients only. The use of cone radius is important to maintain the continuity of the system, such that a consistent comparison is achieved.

Throughout the synchronisation process, however, the most significant uncontrolled variable is the developed drag torque. To be able to completely realise the application of these dimensionless variables it is necessary to quantify the drag torque acting on the synchroniser and then reduce it to a similar dimensionless quantity. It is then logical to divide the drag torque by the operating radius of the cone clutch such that the normalised drag torque can be evaluated directly against dimensionless cone and indexing torques. Thus:

$$
\Pi_{D}=\frac{\sum T_{D}}{F_{A} R_{C}}
$$

\section{Evaluation of dimensionless torques}

The purpose for the development of dimensionless variables describing the torques acting on the synchroniser mechanism is to provide understanding of the influence of certain parameters on the mechanism over a broad range. Here modification to cone and chamfer parameters will enable the further understanding of their specific influence. The cone torque map, Figure 6, has been developed over a range of operating conditions, with the cone angle, $\alpha$, varied from six to ten degrees, and the cone friction 
coefficient, $\mu_{\mathrm{C}}$, varied from 0.06 to 0.16 . In some instances of variation the selected cone angle and friction coefficient breach the friction limit that causes cone lock of $\mu_{\mathrm{C}}>\sin \alpha$. Using this limit the maximum normalised torque is at $\Pi_{\mathrm{C}}=1$.

Variation of the friction and cone angle parameters, as shown in Figure 6, produces substantial variation in the developed torque. Higher torques are achieved using shallower cone angles and higher friction coefficients, but are limited by the friction lock described by Vyrabov (1991). Even with the use of design error angles it is not advised to use parameters that exceed this limit as gradual wear of the cone surface will reduce the error angle leading to eventual lock of the cone.

Referring to equation 26 , when developing the indexing torque map a radius ratio, $\mathrm{R}_{\mathrm{I}} / \mathrm{R}_{\mathrm{C}}$, of 1.2 is used to provide adequate sizing of components for chamfer pitch radius in comparison to mean cone radius. Though a range from $1.1-1.3$ is reasonable if a ratio of these to radii is less than one, this implies that the chamfers are located internally from the cone. For Figure 7 the range of chamfer friction coefficients $\mu_{\mathrm{C}}$ is varied from 0.0 to 0.1 , and chamfer angle, $\beta$, is varied from 55 to 75 degrees.

Evaluation of chamfer torques developed in Figure 7 provides significant demonstration of the ability of the derived torque to match that generated in the cone. Only with the ideal friction case of $\mu_{\mathrm{I}}=0$ is the cone torque limit reached, and for more typical friction coefficients and chamfer angles the dimensionless value is in the band of 0.6 to 0.7 , suggesting that the early unblocking of the synchroniser ring is more a result of ineffective wiping of the cone surface or through other loss of cone friction than through alternate modes. Unlike the variation in the cone torque, increasing friction coefficient reduces the torque developed as it acts against the direction of torque 
developed from the angle, and also plays a smaller role in variation in comparison to the change in chamfer angle.

The most significant issue in developing a drag torque map is that there is considerable variation from gear to gear, depending on both current gear ratio and target gear ratio, making any drag torque map unique to the transmission under consideration. The maps presented in Figures 8 and 9 are for both up and down shifts of all gears, using a range of operating speeds. Thus the peak drag torque can be established over a reasonably wide range of operating conditions. It is important to note here that simulations are performed using an operating temperature of $40^{\circ} \mathrm{C}$, variation in temperatures, particularly use of zero or subzero operating temperatures will have significant effect on the drag torque developed.

Review of the two drag torque maps suggests that, for most applications, single cone synchronisers would be sufficient for overcoming drag torque, particularly as downshifts into first gear at high speeds are very unlikely. However, if consideration of cold start applications is made, where drag losses are significantly higher, the need for double and triple cone synchronisers becomes immediately apparent. Due to the concentrically arranged clutches the drag torque developed in the odd gears is also higher as these gears are engaged with the outer clutch, giving rise to the unexpected order of the dimensionless drag torque.

Significant variation arises in the dimensionless drag torques for up and downshifts as a result of conflicting drag components. The absolute component of drag, that which is linked to the gear speed, acts only against this speed. Whilst the drag torque associated with the relative speed in the wet clutch pack can act with or against the 
absolute drag torque. This is clearly demonstrated in Figures 8 and 9 where normalised drag is minimal at low speeds as the two torques are apposed, but for similar speed upshifts drag torque is substantially lower.

The comparison of Figure 8 and 9 with Figure 6 demonstrates that there is ample advantage for cone torque overcoming drag torque in most applications whilst under standard operating conditions. Generally speaking this is also true for the indexing torques, Figure 7, however poor selection of chamfer angle suggests that there could be significant resistance to unblocking or indexing of the mechanism under certain conditions. Obviously the higher drag developed in lower gears as a result of the differential speeds will increase the resistance load on indexing torque, thus more acute chamfer angles are required for lower gears.

As a comparative example of the different influence of up and down shift drags when selecting parameters one can compare several gear selections. For fourth gear, in both up and downshifts at normal operating temperatures dimensionless drag is below 0.4 for the entire operating range. Beginning with the cone torque requirements, to minimise synchronisation time the maximum dimensionless torque is chosen given the friction limit. So with a friction coefficient of 0.12 , the maximum friction angle is about $7.5^{\circ}$. Considering nominal operating conditions the dimensionless indexing torque must be less than the cone torque but greater than the dimensionless drag torque. If the friction coefficient is assumed to be 0.06 , then, for a dimensionless value of 0.7 the chamfer angle will be at $57^{\circ}$. By comparison, a first gear synchroniser, at peak engine speeds, already has a dimensionless torque value for downshifts at $80 \%$ of the dimensionless cone torque, thus multicone synchronisers are most definitely required under even 
standard operating conditions. Furthermore, with these conditions it may be necessary to increase the pitch diameter of the chamfers, or choose more aggressive chamfer angles to overcome this drag torque. Alternate solutions must consider the operation of the wet clutch through evacuation of the open clutch pack of transmission fluid during synchronisation under adverse operating conditions.

In this section, dimensionless torques derived in Section 4 have been studied to investigate the influence of specific design variables on the design and operation of synchroniser mechanisms. Conducive to this is the relating of cone and chamfer torques to the less well understood drag torque, where utilisation of the operating radius of the cone and peak hydraulic force provides a simple method for evaluating the drag torque over a wide range of operating conditions. To see the effects of varying the design parameters of the synchroniser mechanism, however, a parameter study of the design can be performed varying each of the four design variables independently.

\section{Parameter study of mechanism}

Confirmation of the results demonstrated by the dimensionless analysis of the synchroniser mechanism, particularly when considering the four selected design variables is best realised through the performance of a parameter study of the mechanism. Thus the following section details the results achieved through variation of these four variables independently. It is also possible to change the radius ratio and actuation pressure to further extend the study. However the variation is more obvious, particularly as increasing the radius ratio will increase the indexing torque and increasing the pressure 
will only reduce the actuation time. To maintain consistency of results the following parameters are used as general constants whilst the target parameter is changed.

Table 1: Synchroniser and Gear Properties

\begin{tabular}{l|c}
\hline \multicolumn{1}{c|}{ Parameter: } & $4^{\text {th }}$ Gear \\
\hline Reflected inertia of target gear $\left(\mathrm{kg} \cdot \mathrm{m}^{2}\right)$ & 0.0097 \\
Mean cone diameter $(\mathrm{mm})$ & 95 \\
Cone angle $\alpha\left(^{\circ}\right)$ & 7 \\
Cone friction coefficient $\mu_{\mathrm{C}}$ & 0.1 \\
Pitch diameter of chamfers $(\mathrm{mm})$ & 120 \\
Chamfer angle $\beta\left(^{\circ}\right)$ & 60 \\
Chamfer friction coefficient $\mu_{\mathrm{I}}$ & 0.1 \\
Maximum sleeve force $\mathrm{F}_{\mathrm{A}}(\mathrm{N})$ & 350 \\
\hline
\end{tabular}

The independent variation of each of these variables in simulation can provide further insight into the influence exerted on the mechanism by different design parameters. It should be noted that some of these variations will deliberately breach design limitations set out at the beginning of this chapter. To maintain continuity across each variation the sleeve displacement alone will be used to demonstrate the results.

First target is the variation in synchroniser cone angle, with results shown in Figure 10, with the friction coefficient of 0.12 simulations with cone angles of $6^{\circ}$ and $7^{\circ}$ are below the friction limit of $\mu_{\mathrm{C}}>\sin (\alpha)$. As prescribed in equation 26, it would be expected that a decrease in cone angle results in an increase cone torque, hence decreasing the synchronisation time. Results of the simulation demonstrate decreasing the cone angle has the effect of reducing synchronisation time in a reasonably linear form, 
with a one degree decrease correlating to a decrease in synchronisation time of less than 10ms. This is countered by the change in angle increasing the oil squeezing resistance during initial displacement, though the duration of this change is much smaller than $1 \mathrm{~ms}$ per degree of cone angle.

Secondly, the cone friction coefficient is varied through a range of $\mu_{\mathrm{C}}=0.06$ to $\mu_{\mathrm{C}}=0.16$, with the cone angle set to $7^{\circ}$ simulations with friction coefficients above $\mu_{\mathrm{C}}=0.10$ breach the friction limit convention. The results presented in Figure 11 demonstrate a highly nonlinear change to the increase in cone friction, with the influence of increasing $\mu_{\mathrm{D}}$ by a factor of 0.2 having less and less significance above $\mu_{\mathrm{C}}=0.10$. However, it is clearly demonstrated that the use of higher friction coefficients is more desirable as compared to lower values, particularly as limitations to cone angles are included. These simulation results are consistent with the dimensionless friction results presented in Figure 6, where modification to friction coefficient is demonstrated as nonlinear but cone angle change produces linear variation in dimensionless cone torque.

Next, variation of chamfer angle, $\beta$, is simulated, in the range of $55^{\circ}$ to $75^{\circ}$ degrees. Typically $\beta$ may be up to $65^{\circ}$, but more acute angles may be necessary given the nature of drag torque in the wet clutch. The variation in this parameter demonstrates a trade off in the mechanism design, where, if the chamfer toque is to be increased at a particular pitch radius a longer sleeve displacement is required. This equates to about one millimetre for every 5 degree change, and can add significantly to the overall envelop of the transmission design. The results show that with continued increase to the chamfer angle the overall process time is extended, which is somewhat offset by the reduced displacement. This is a result of lower blocking torques increasing the ring unblocking 
and hub indexing times. The selection of chamfer angle then brings about a peculiar balance, where overall engagement time can be minimised by either using a blunt angle with a more compact design, but exposing the mechanism to the potential for blockout at the ring. Or by using an acute chamfer angle to increase the blocking and indexing torques at the risk of causing premature unblocking of the ring.

Finally, the chamfer coefficient of friction is modified, between the ideal of $\mu_{\mathrm{I}}=0$ and $\mu_{\mathrm{I}}=0.1$, Figure 13 presents the sleeve displacement for the entire process. This demonstrates that the friction coefficient has the least significance of influences for each design parameter. Where, over the entire range of variation, reduction in simulation time varies by approximately $3 \mathrm{~ms}$. Thus it is favourable to minimise the chamfer friction but its reduction has minimal impact on the actuation of the system in terms of response times. The primary benefit is rather associated with the increased indexing torque as the associated friction loss is minimised.

Thus the comparison of chamfer variables leads to the conclusion that the chamfer friction coefficient is of minimal importance in comparison to the chamfer angle. This again verifies the dimensionless results shown in Figure 7. These results implied that the significance in changing chamfer angle greatly surpasses modification to the friction coefficient.

\section{Conclusions}

Core to the development and application of conventional synchroniser as a means for gear shifting in the dual clutch transmission is the demonstration that the mechanism is reliable under a broad range of operating conditions. Demonstration of the influence of 
drag torque on the synchronisation process is key to the application of the mechanism in a reliable manner. Through the normalisation of key torque equations and the simulated drag torque on the mechanisms it became possible to develop a depiction of the variation of drag acting on the mechanism under a wide range of operating speeds. The result of this analysis suggests that with reasonable care on parameter selection most standard synchroniser designs should be capable of providing reliable speed synchronisation. However, issues arise with ring unblocking if there is poor selection of chamfer torque, particularly under adverse operating conditions, such as high speed or low temperature.

Investigation of the key design parameters of the synchroniser mechanism suggests that the time duration of speed synchronisation can be significantly reduced through the application of large friction coefficient or smaller cone angles. However, when breaching the torque ratio limit it would become impossible to disengage the cone, thus the limit is maintained. The friction coefficient variable in the indexing and blocking chamfers has minimal influence on the engagement of the mechanism, whilst the use of acute chamfer angles will reduce the engagement time without significantly increasing the likelihood of early unblocking of the mechanism.

\section{Acknowledgments}

The financial support of this work by the Australian Research Council (ARC LP0775445) and the University of Technology, Sydney, is gratefully acknowledged.

\section{References}


Abdel-Halim, N., Barton, D., Crolla, D. \& Selim, A. (2000), 'Performance of Multicone Synchronizers for Manual Transmissions', Proceedings of the Institution of Mechanical Engineers, Part D: Journal of Automobile Engineering, vol. 214, pp. $55-65$

Anderson, N.E. \& Loewenthal, S.H. (1980) 'Spur Gear System Efficiency at Part and Full Load', NASA Technical Paper 1622

Changenet, C., Oviedo-Marlot, X. \& Velex, P. (2006) 'Power loss predictions in geared transmissions using thermal networks-application to a six speed manual gearbox' Transactions of the ASME: Journal of Mechanical Design, vol. 128, no. 3, pp. $618-625$

Changenet, C. and Velex, P., (2007) 'A Model for the Prediction of Churning Losses in Geared Transmissions - Preliminary Results', Transactions of the American Society of Mechanical Engineers, Vol. 129, Iss. January, pp. 128-133

Diab, Y., Ville, F., Velex, P. and Changenet, C., (2004) 'Windage losses in high speed gears - preliminary experimental and theoretical results', Journal of Mechanical Design, Vol. 126, pp. 903-908.

Dogan, S. N., Ryborz, J., Bertsche, B., (2004) 'Rattling and clattering noise in automotive transmissions - simulations of drag torque and noise', Transient Processes in Tribology, Lyon France, pp. 109-121

Eastwick, C. N. and Johnson, G., (2008) 'Gear Windage: A Review', Journal of Mechanical Design, Vol. 130, Iss. March

Goetz, M. (2005) Integrated Powertrain control for Twin Clutch Transmissions, PhD Thesis, University of Leeds, Leeds, United Kingdom 
Harris, T. A., Rolling Bearing Analysis, (1966) John Wiley \& Sons, Inc.

Heingartner, P. \& Mba, D. (2005), 'Determining Power Losses in Helical Gear Mesh', Gear Technology, No. 3, pp. 32-37.

Hoshino, H. (1999), Analysis on Synchronisation Mechanism of Transmission, SAE Technical Paper no: 199-01-0734

Jeyakumaran, J. \& Zhang, N. (2005) 'Friction induced Vibration of Clutches in an Automatic Transmission', Proceedings of the Asia Pacific Vibration Conference, Langkawi, Malaysia, pp. 174-179

Kelly, D. \& Kent, C. (2000) 'Gear shift quality improvement in manual transmissions using dynamic modeling, FISITA world automotive congress, Paper No. F2000A126

Kim, J., Park, S., Seok, C., Song, H., Sung, D., Lim, C., Kim, J. and Kim, H. (2003), 'Simulation of the shift force for a manual transmission', Proceedings of the Institution of Mechanical Engineers, Part D: Journal of Automobile Engineering, vol. 217 , pp. $573-581$

Kirschstein, S. 2007, 'The Impact of Launch Control on the Vibration Behavior of a Dual Clutch Transmission Powertrain', VDI Berichte, vol. 1971, pp. 197-217.

Kulkarni, M., Shim, T. \& Zhang, Y. (2007), 'Shift Dynamics and Control of Dual-Clutch Transmissions', Mechanism and Machine Theory, vol. 42, pp. 168-182.

Lechner, G. \& Naunheimer, H. (1999), Automotive Transmissions - Fundamentals, Selection, Design and Application, 1st edn, Springer-Verlag, Germany 
Lei, Y., Wang, J. \& Ge, A. (2006) "Research on Control strategies of Double Clutch Transmission Based on System Simulation", FISITA world automotive congress, Paper No. F2006P041

Liu, Y. \& Tseng, C. (2007) 'Simulation and Analysis of Synchronisation and Engagement on Manual Transmission Gearbox', International Journal of Vehicle Design, vol. 43, no. 1-4, pp. 200-220

Lovas, L., Play, D., Marialigeti, J. \& Rigal, J. (2006), 'Mechanical behavior simulation for synchromesh mechanism improvements', Proceedings of the Institution of Mechanical Engineers Part D: Journal of Automobile Engineering, vol. 220, pp. 919-945.

Manring, N.D. (2005) Hydraulic control systems, 1st edn, John Wiley \& Sons, USA

Paffoni, B., Progri, R., Gras, R. \& Blouet, J. (1997), 'The hydrodynamic phase of Gearbox synchromesh operation: the influence of radial and circumferential grooves', Proceedings of the Institution of Mechanical Engineers, Part J: Journal of Engineering Tribology, vol. 211, pp. 107-116.

Razzacki, S.T. (2004) Synchroniser Design: A Mathematical and Dimensional Treatise, SAE Technical Paper No. 2004-01-1230

Razzacki, S.T. \& Hottenstein, J. (2007) Synchroniser Design and Development for Dual Clutch Transmission (DCT), SAE Technical Paper No. 2007-01-0114

Schilchting, H., (1979) Boundary layer theory, McGraw-Hill Classic.

Socin, R.J. \& Walters, L.K. (1968) Manual Transmission Synchronizers, SAE Technical Paper No. 680008 
Stringer, J. (1976), Hydraulic systems analysis, an introduction, 1st edn, Macmillan Press Ltd, London, UK

Vyrabov, R.V. (1991), 'Friction in a cone clutch and in a friction drive with wedge type bodies', Transactions of the ASME, Journal of tribology, vol. 113, no. 5, pp. 681688

Walker, P.D., Zhang, N., Tamba, R. \& Fitzgerald, S. (2009), Synchroniser modeling with application specific to the dual clutch transmission, Proceedings of the Asia Pacific Vibration Conference, Paper no. 088

Watechagit, S. \& Srinivasan, K. (2003), Modeling and Simulation of Shift Hydraulic System for a Stepped Automatic Transmission, SAE Technical Paper No. 2003$01-0314$

Yuan, Y., Liu, E., Hill, J. and Zou, Q., (2007) An Improved Hydrodynamic Model for Open Wet Transmission Clutches, Journal of Fluids Engineering, Vol. 129, pp. 333-337.

Zhang, Y., Chen, X., Zhang, X., Jiang, H. \& Tobler, W. (2005), 'Dynamic Modeling and Simulation of a Dual-Clutch Automated Lay-Shaft Transmission', Transactions of the American Society of Mechanical Engineers, vol. 127, pp. 302-307.

\section{Appendix 1}

To develop the pressure model of the two hydraulic cylinders presented in Figure 1 , begin with the differential equation of a compressible fluid, where $\mathrm{Q}$ is the flow rate, $\mathrm{V}$ is the fluid volume, $\mathrm{P}$ is pressure, and $\beta$ is the bulk modulus

$$
Q=\frac{V}{B} \frac{d P}{d t}
$$


Arranged to make pressure the subject

$$
\int d P=\int \frac{Q \cdot B}{V} d t
$$

With motion of the sleeve the volume of the cylinder will change, thus volume is not constant, or:

$$
\int d P=\int \frac{Q \cdot B}{V_{0}+d V} d t
$$

It is assumed that the bulk modulus, $\beta$, is constant, so the relevant flow rates are required for inflow from the orifice, rate of change of cylinder, and leak flow out of the control volume. $\mathrm{Q}$ is therefore:

$$
Q=Q_{\text {Orifice }}+Q_{\text {Volume }}+Q_{\text {Leak }}
$$

Or, based on equations for sharp edged orifice and annular orifice presented in Stringer (1976), and the rate of change in volume of the cylinder:

$$
Q=C_{D} \pi \frac{D_{C V 1}^{2}}{4} \sqrt{P_{S}-P_{C V 1}}-A_{S} \frac{d X_{S}}{d t}-C_{D} \pi D_{S} c_{R S} \sqrt{P_{C V 1}-P_{E X}}
$$

Equation 31 is then substituted into equation 29 to give Equations 1 and 2, repeated below, note that the sign difference for the volume rate of change is required as the piston heads move in opposing directions for the two cylinders:

$$
P_{C V 1}=\int \frac{B}{V_{0}+d V}\left(C_{D} \pi \frac{D_{C V 1}^{2}}{4} \sqrt{P_{S}-P_{C V 1}}-A_{S} \frac{d X_{S}}{d t}-C_{C V 1} \pi D_{S} c_{R S} \sqrt{P_{C V 1}-P_{E X}}\right) d t
$$

\section{Appendix 2}

Assuming that over the realignment period the variation in drag torque is negligible, to determine an approximate unblocking time: 
$\delta=\dot{\theta}_{S} t+0.5 \ddot{\theta}_{S} t$, which can be arranged as $t=\sqrt{\frac{2 \delta}{\ddot{\theta}_{S}}}$

$(32 \mathrm{a} \& b)$

Applying Newton's second law, $\sum M=I \alpha$

$I_{F W} \ddot{\theta}_{S}=T_{D}+T_{B}$, or $\ddot{\theta}_{S}=\frac{T_{D}+T_{B}}{I_{F W}}$

$(33 \mathrm{a} \& b)$

Combining these equations:

$t_{B}=\sqrt{\frac{2 \delta I_{F W}}{T_{D}+T_{B}}}$ 


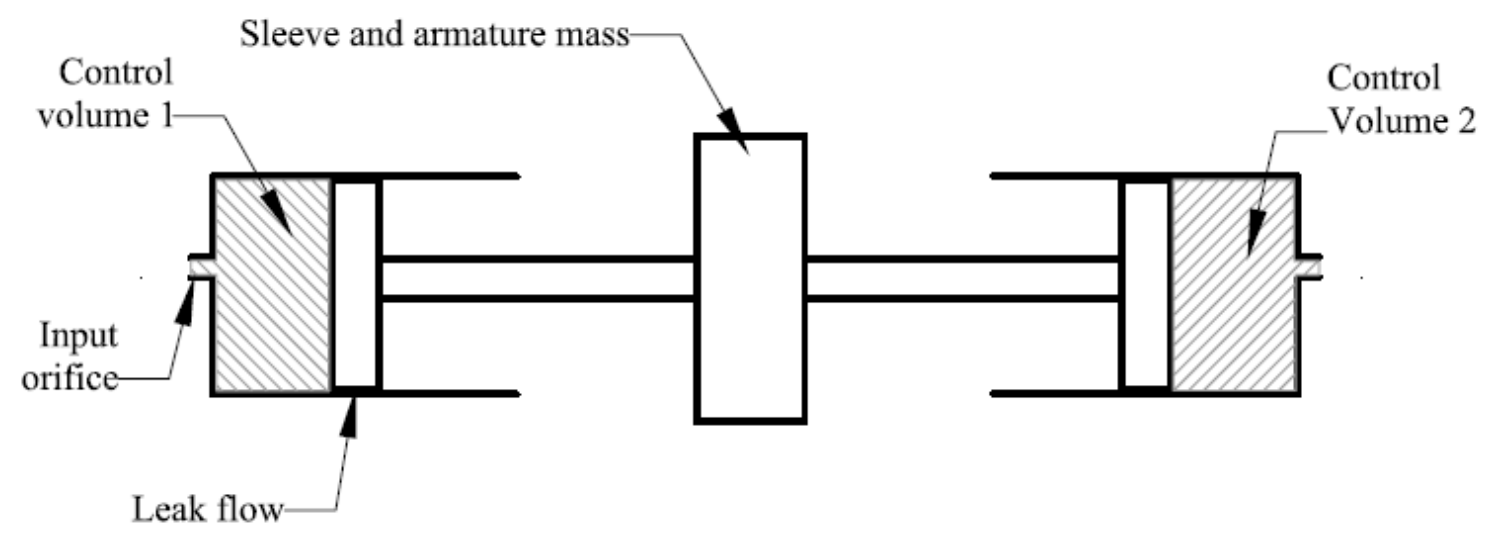

Figure 1: Synchroniser hydraulic unit, with control pressure to the input orifice taken from on/off solenoids actuated with a step input.

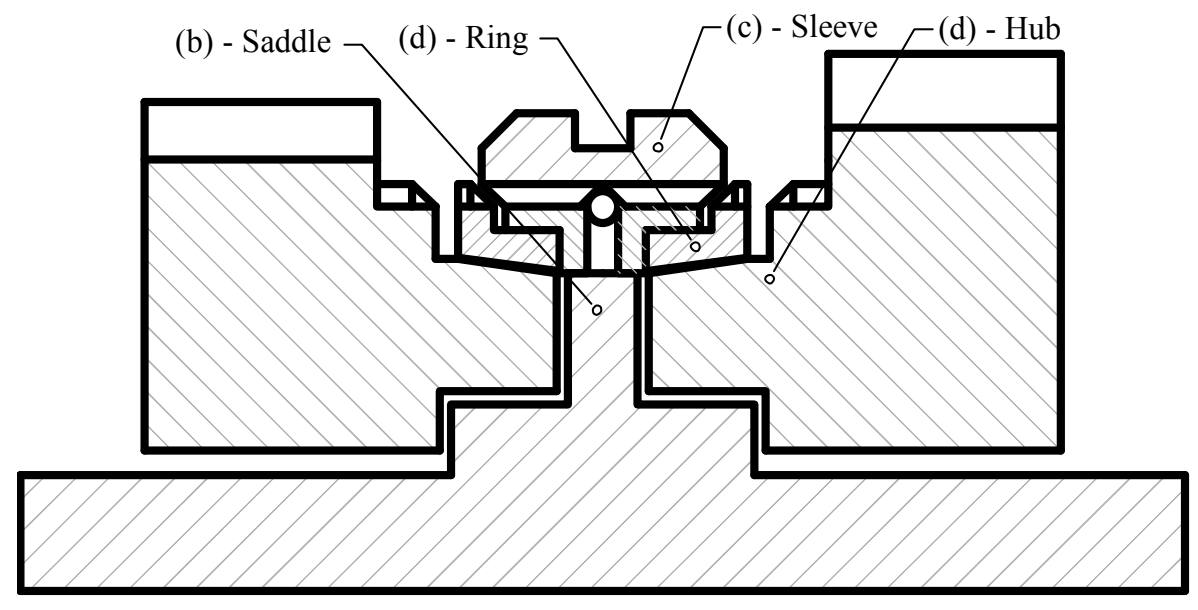

Figure 2: Cross section of a typical synchroniser 

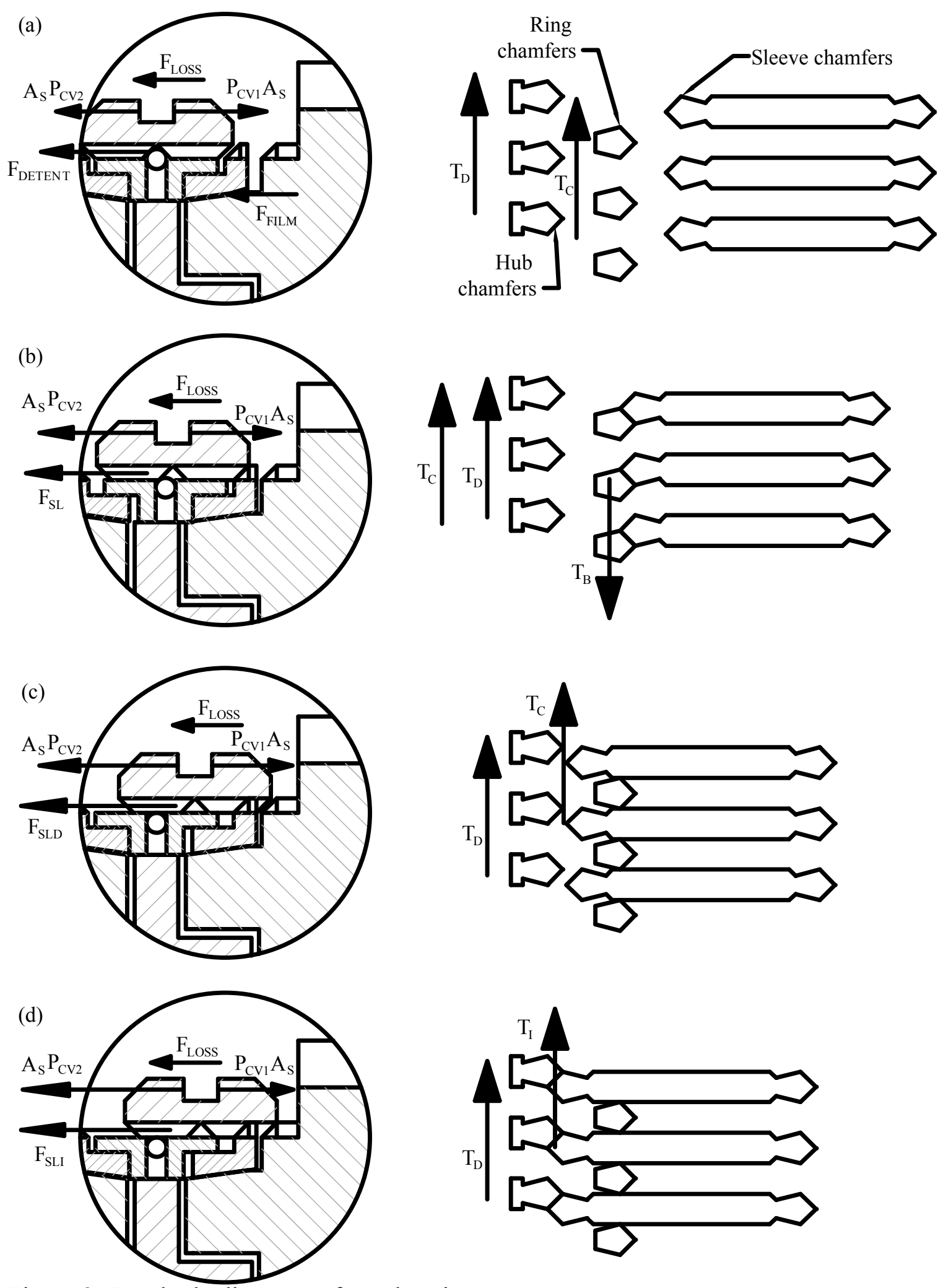

Figure 3: Free body diagrams of synchroniser engagement 


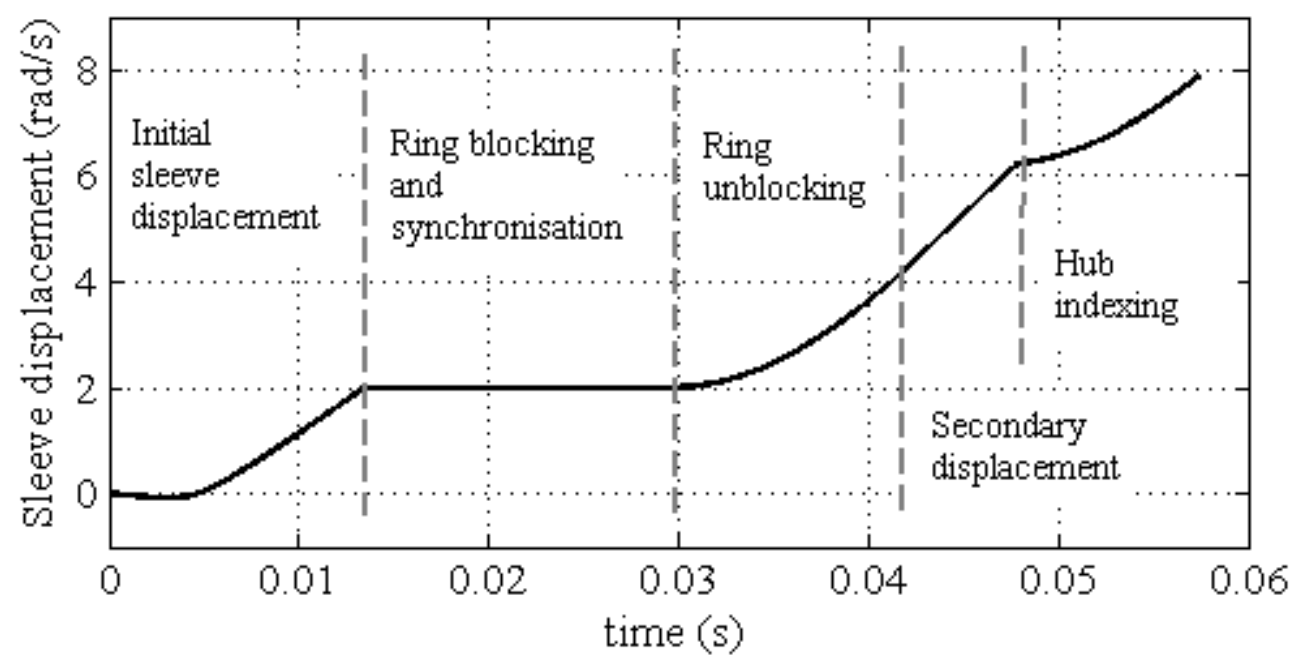

Figure 4: Synchronisation process breakdown

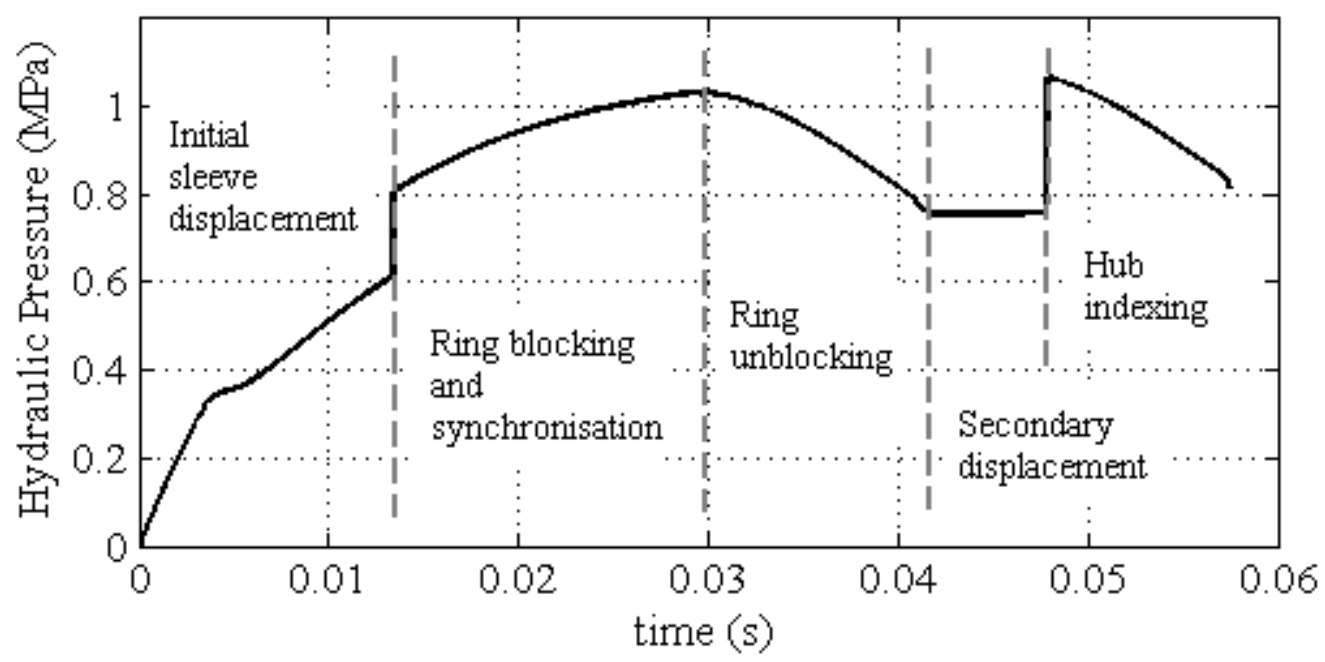

Figure 5: Control cylinder pressure response 


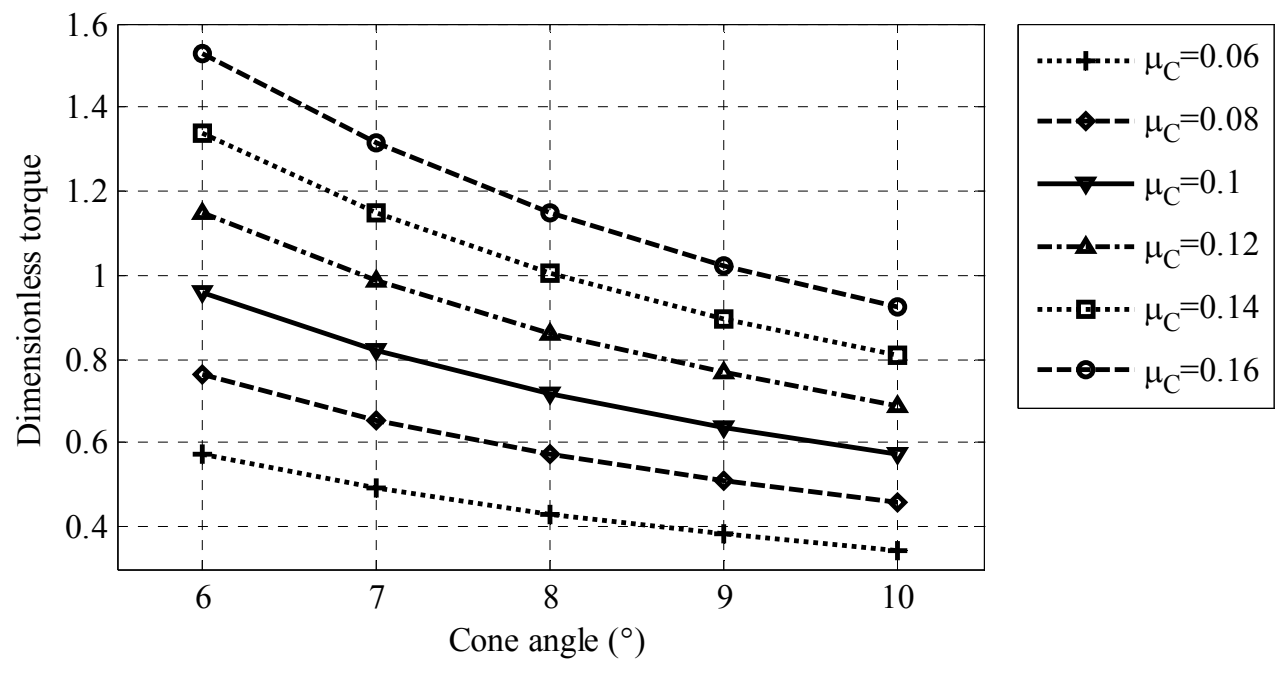

Figure 6: Dimensionless cone torque

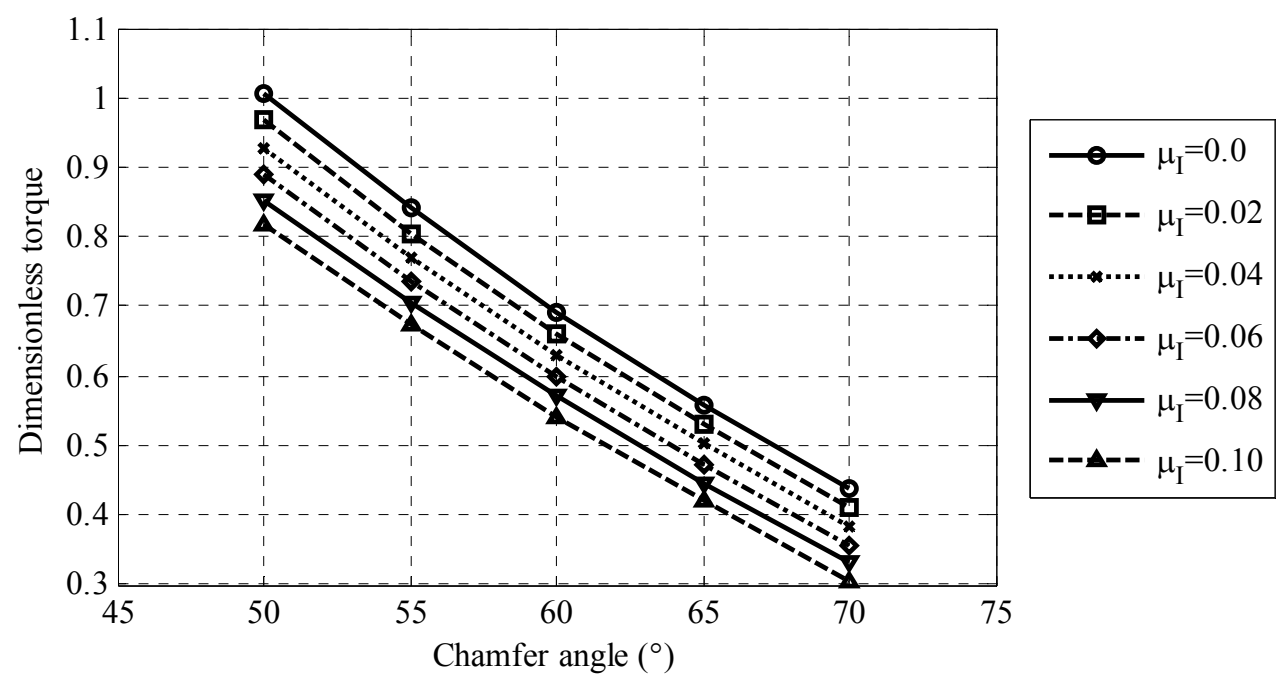

Figure 7: Dimensionless blocking/indexing torque 


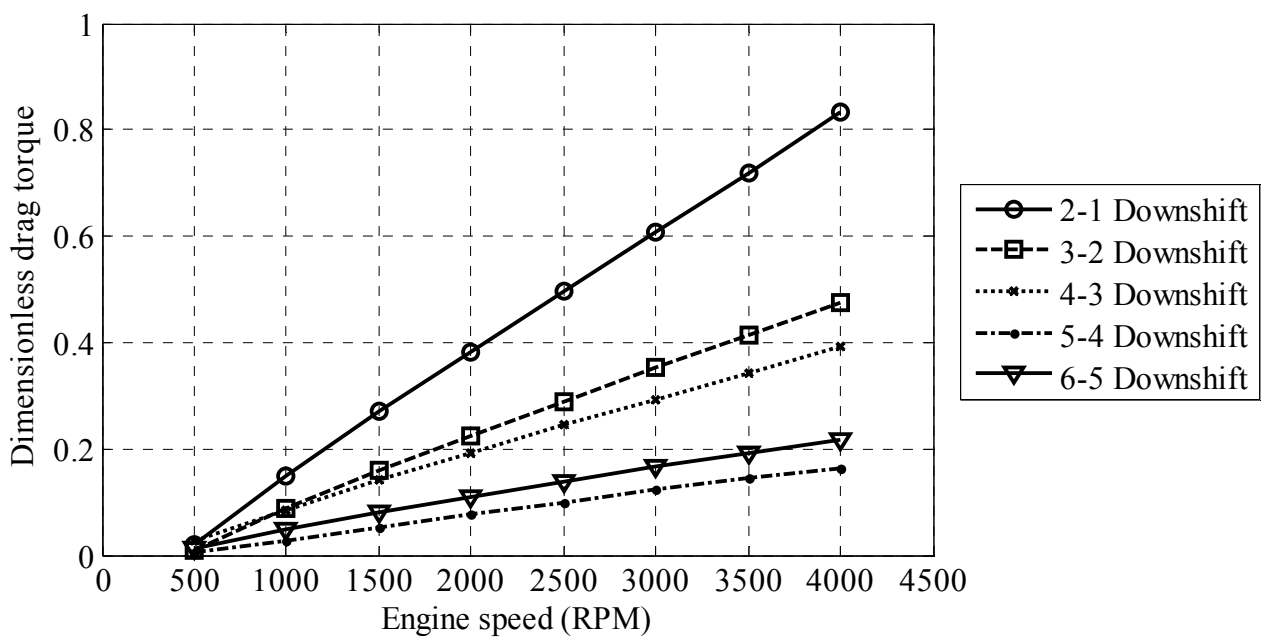

Figure 8: Dimensionless drag torque for downshifts

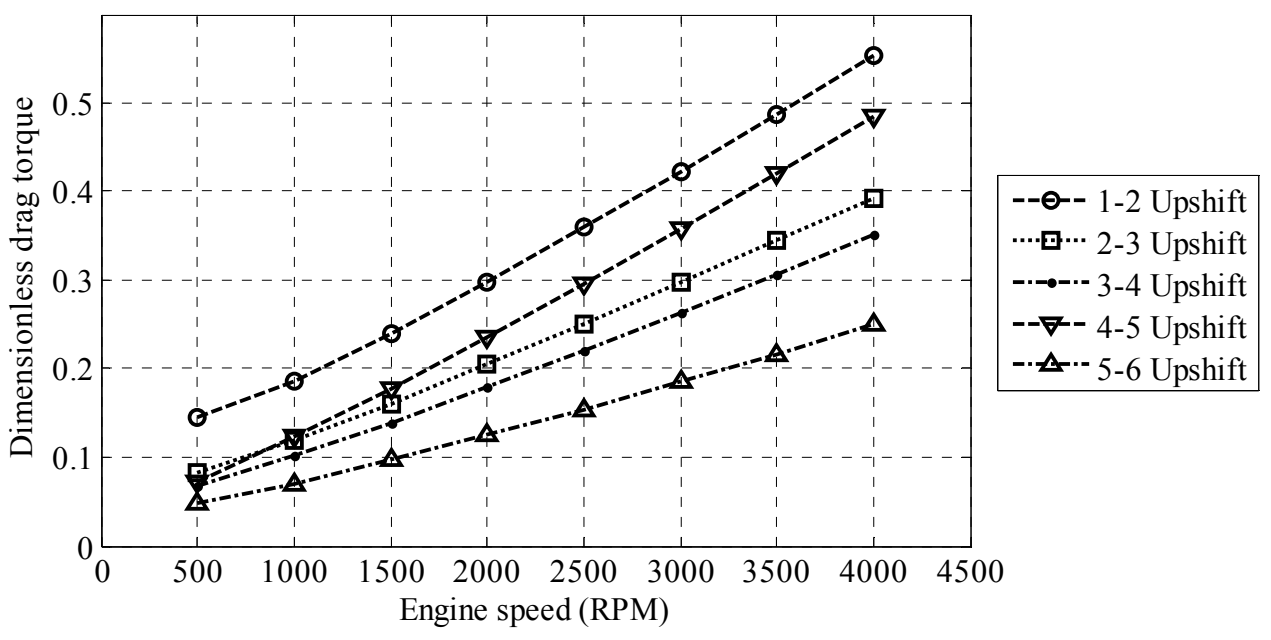

Figure 9: Dimensionless drag torque for up shifts 


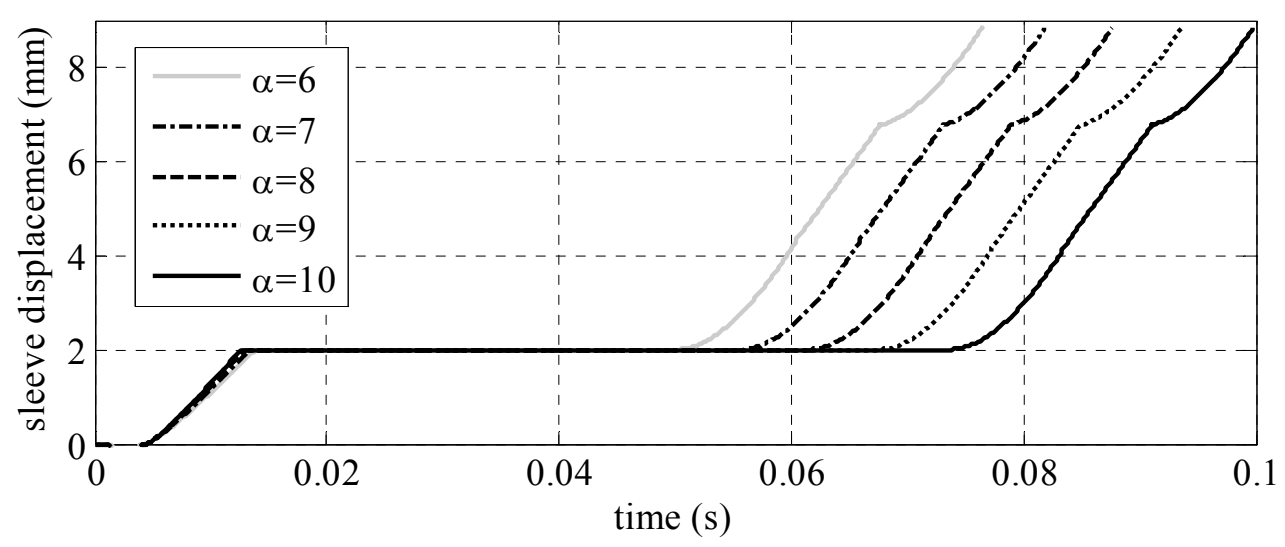

Figure 10: Parameter modification to cone angle

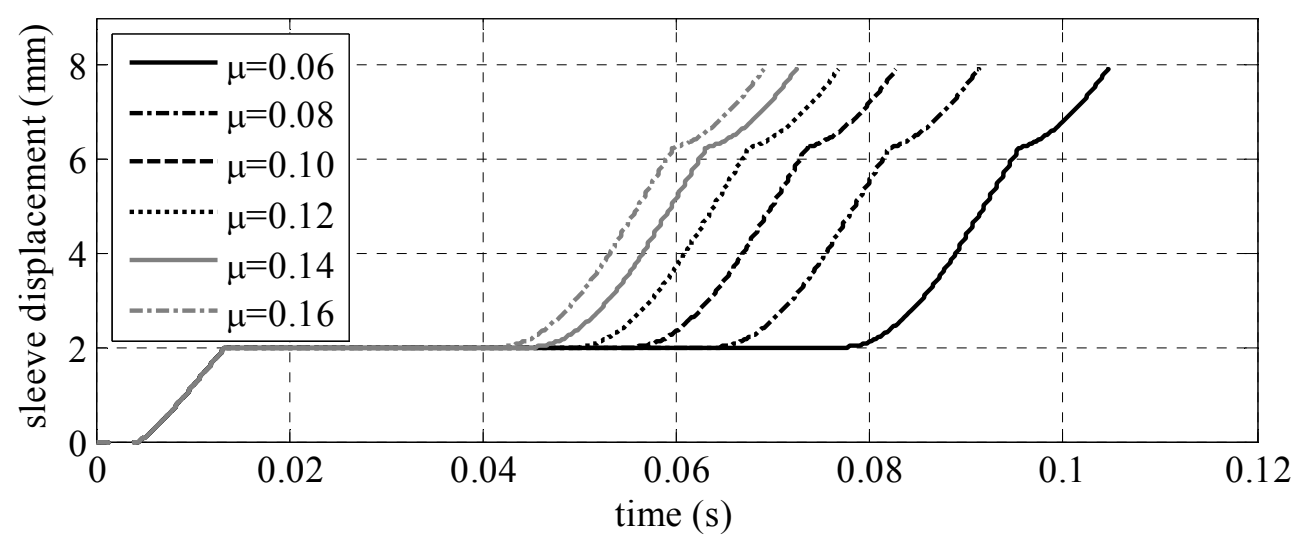

Figure 11: Parameter modification to friction coefficient 


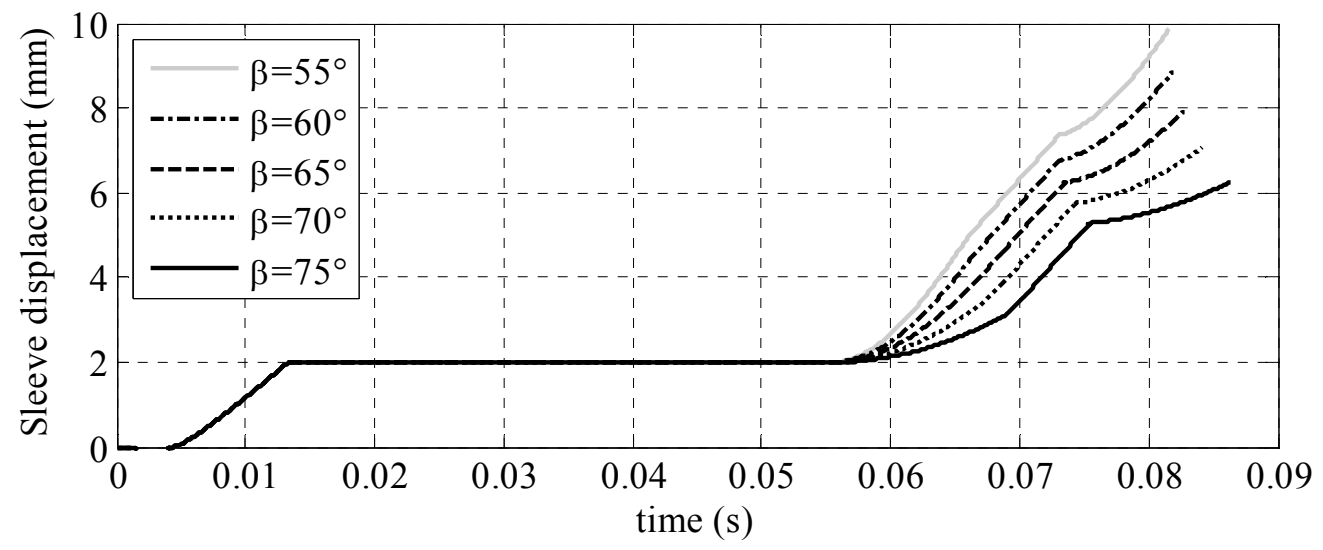

Figure 12: Chamfer angle parameter modification

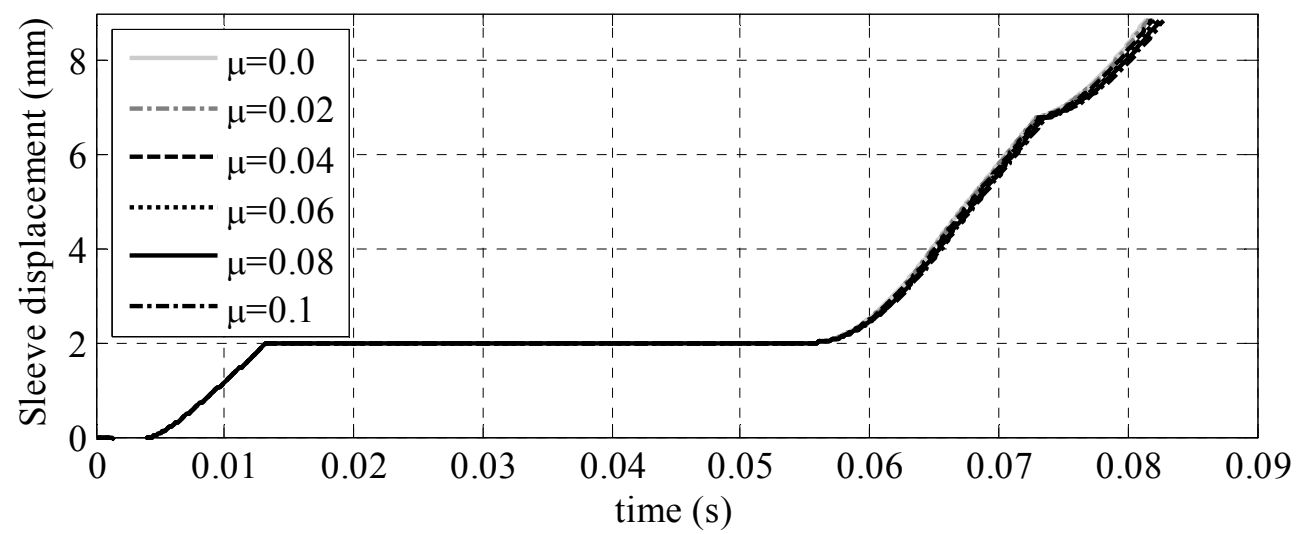

Figure 13: Parameter modification to chamfer friction 